EXTENDED REPORT

\title{
Systemic carboplatin for retinoblastoma: change in tumour size over time
}

\author{
D H Abramson, S D Lawrence, K L Beaverson, T C Lee, I S Rollins, I J Dunkel
}

Br J Ophthalmol 2005;89:1616-1619. doi: 10.1136/bjo.2005.072033

See end of article for authors' affiliations

.....................

Correspondence to:

David H Abramson, MD,

70 East 66th Street, New

York, NY 10021, USA;

DHAMD@aol.com

Accepted for publication 1 August 2005
Background/aim: Chemotherapy for intraocular retinoblastoma is used to shrink individual retinal tumours to a size amenable to focal treatments. Quantitative data regarding retinal tumour response following treatment with primary systemic carboplatin are reported.

Methods: Changes in area and largest basal diameter of tumours that were exposed to carboplatin, had no concomitant focal treatment, and had digital funduscopic photography performed before and after treatment, were measured. Response was evaluated.

Results: 36 tumours were measured following one treatment: $34 / 36$ (94.4\%) responded, with a $37.1 \%$ mean decrease in area (median $=37.0 \%$; range $4.0 \%-76.7 \%$ ). Mean reduction in basal diameter was 21.3\% (med=21.0\%; -7.9\%-52.5\%). 20 tumours were treated with a second cycle: 15/20 (75.0\%) responded. Mean decrease in area was $17.8 \%$ ( $\mathrm{med}=15.3 \% ;-7.0 \%-49.7 \%)$. The mean cumulative decrease in area after two treatments was $55.1 \%$ (med $=56.2 \% ; 33.0 \%-74.5 \%$ ). Mean cumulative reduction in basal diameter was $33.6 \%$ ( $\mathrm{med}=33.6 \%$; 10.9\%-53.2\%). 12 tumours were treated with a third cycle: $3 / 12(25.0 \%)$ responded, $8 / 12$ were stable, and one progressed. Mean decrease in area was $5.4 \%$ (med $=7.2 \% ;-17.7 \%-20.6 \%$ ). Cumulative decrease in area after three treatments was $58.1 \%$ $(\mathrm{med}=57.3 \%$; $34.8 \%-77.2 \%)$. Mean cumulative reduction in basal diameter was $38.8 \%$ (med $=38.2 \%$; $19.1 \%-54.1 \%)$.

Conclusions: Carboplatin caused measurable shrinkage of retinoblastoma tumours. Response was greatest following the initial treatment and decreased with subsequent treatments.
$\mathrm{P}$ rimary chemotherapy is often used to treat intraocular retinoblastoma, but when used alone, it is rarely curative. For select eyes, chemotherapy may be used to shrink retinal tumours (chemoreduction) to a size cured by the application of focal techniques such as cryotherapy, brachytherapy, and laser photocoagulation. The protocol most commonly used to achieve chemoreduction is a combination of vincristine, etoposide, and carboplatin (VEC). Ciclosporin may be added to the regimen with the goal of decreasing multidrug resistance. ${ }^{1}$ One's choice of drugs, dosage and treatment schedule must weigh patient/ tumour response against the risks and/or complications of treatment. Known risks of chemotherapy treatment exist; including, haematological (myelosuppression) and gastrointestinal distress and infection. ${ }^{2}$ Furthermore, eyes remain at risk for new retinal tumour development following treatment with chemotherapy. ${ }^{3}$ Known complications of chemoreduction plus focal therapies also exist; including, rhegmatogenous retinal detachment in the setting of chemoreduction plus cryotherapy, ${ }^{4}$ and focal iris atrophy, peripheral focal lens opacity, retinal traction, retinal vascular occlusion, and transient localised serous retinal detachment in the setting of thermotherapy. ${ }^{5}$ The development of additional cancers has been reported following treatment with chemotherapy. Secondary myelodysplastic syndrome/ acute myelogenous leukaemia is a rare, but devastating, side effect of a number of chemotherapeutic agents, but has been particularly associated with etoposide. ${ }^{6}$ Known patient/ tumour response data to chemoreduction techniques are most often defined by outcome variables associated with ocular and patient survival, as well as avoidance of external beam radiotherapy (EBR). The most consistent correlate for success is extent of intraocular disease; Reese-Ellsworth group (RE) I-III eyes usually avoid EBR and enucleation, while RE group IV-V eyes have lower success rates. ${ }^{27}$ Several studies have examined the effects of systemic chemotherapy on intraocular retinoblastoma in the setting of concomitant focal therapies. ${ }^{8-13}$ These studies accurately reflect common clinical practice, but when reporting outcome data, their methodology does not allow for the evaluation of any isolated effect of chemotherapy on tumour morphology.

A few studies have attempted to rigorously quantify the change in retinal tumour size following primary treatment with chemotherapy by means of serial measurements, pretreatment and post-treatment. ${ }^{14-16}$ These published studies focus on response after two and three drug regimens and measure tumour dimensions by various methods. All report notable shrinkage after a single cycle of chemotherapy, with decreased continued reduction following successive doses. We are unaware of any study to date that has quantified reduction in tumour size as a function of a single agent, systemic chemotherapy, in the absence of additional treatment(s).

\section{PATIENTS AND METHODS}

We performed a retrospective study of retinoblastomas that met the following criteria: (1) they were identified in patients diagnosed with intraocular bilateral or unilateral retinoblastoma, (2) they were exposed to primary carboplatin only, and (3) they had adequate digital fundus photographs (RETCAM) taken before and after exposure to systemic carboplatin. Thirty six tumours in 27 eyes of 21 patients who were treated 1994 to March 2004 at the Ophthalmic Oncology Center of New York Presbyterian Hospital (NYPH) and Memorial Sloan-Kettering Cancer Center (MSKCC) fulfilled

Abbreviations: $\mathrm{CBC}$, complete blood count; $\mathrm{CTT}$, chemothermotherapy; dd, disc diameter; EBR, external beam radiotherapy; RE, ReeseEllsworth; TTT, transpupillary thermotherapy; VEC, vincristine, etoposide, and carboplatin 


\begin{tabular}{ll} 
Table 1 & Patient and tumour demographics. \\
\hline Variable & No (\%) \\
\hline Sex & $12(57 \%)$ \\
Male & $9(43 \%)$ \\
Female & $9(0.75-28.5)$ \\
Mean age at diagnosis (months) (range) & $15(71 \%)$ \\
Disease involvement* & $6(29 \%)$ \\
Bilateral & $8(22 \% 0$ \\
Unilateral & $22(61 \%)$ \\
Tumour location & $6(17 \%)$ \\
Macular & \\
Macular-equator & Equator-ora serrata \\
\hline *At diagnosis and follow up (mean follow up= months).
\end{tabular}

the inclusion criteria. This study protocol was approved by NYPH-Weill Cornell Medical College's institutional review board (Protocol 0404-277).

Collected patient data included sex, age at diagnosis, disease laterality, location of tumour(s), and all subsequent treatments. Tumour location was classified according to its zone: macular (between the superior and inferior temporal arterioles), macular-equator (area of the retina between the macular zone and the equator), and equator-ora serrata (anterior to the equator). Tumours were excluded from analysis if they received any treatment before administration of systemic carboplatin. Tumours were measured using RETCAM imaging software that calculated area from a tracing of the outer margins of the base of the tumour. Two observers conducted independent area measurements of each tumour and the results were averaged into one final data set. The largest basal diameter of each tumour was measured from the RET-CAM images at baseline and following each cycle of chemotherapy. Tumours were censored from the study when additional therapeutic modalities were applied.

We defined tumour response in two ways: (1) the percentage reduction in area and percentage reduction in largest basal diameter were calculated for each treatment (average follow up 3-4 weeks), and (2) based on area measurements, a tumour was described, per treatment, as responsive $(>10 \%$ reduction in area), progressive $(>10 \%$ increase in area), or stable $(<10 \%$ decrease or increase in area).

The diagnosis of retinoblastoma was made on the basis of dilated fundus examination under anaesthesia using indirect ophthalmoscopy, scleral depression, and RET-CAM imaging. Intravenous (IV) carboplatin was administered within days to weeks of diagnosis, at a dose of $18.7 \mathrm{mg} / \mathrm{kg}$ for children under $12 \mathrm{~kg}$ in weight and $560 \mathrm{mg} / \mathrm{m}^{2}$ for children $12 \mathrm{~kg}$ or more. All patients receiving IV carboplatin had a history and physical, complete blood count (CBC), and serum chemistry

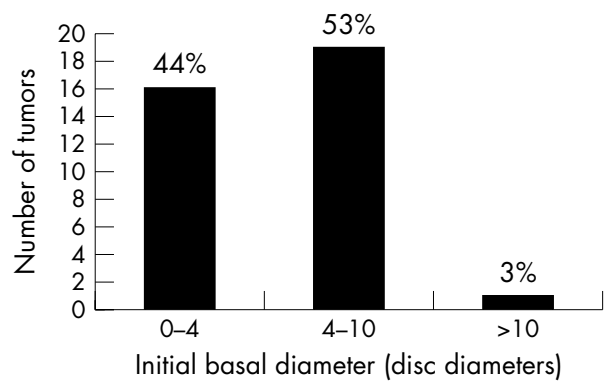

Figure 1 Initial size of tumour at the time of first treatment with systemic carboplatin. The largest basal diameter for each tumour (1 disc diameter $=1.5 \mathrm{~mm}$ ) was measured using RET-CAM imaging.

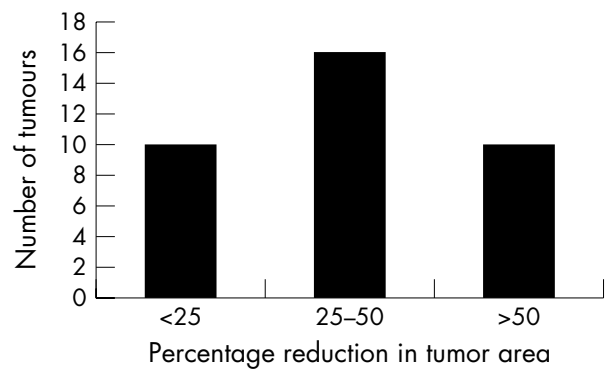

Figure 2 Percentage reduction in tumour area after one initial treatment with systemic carboplatin, stratified.

performed in order to evaluate systemic effects of the drug. Ophthalmological examinations were performed at 3-4 week intervals (average).

\section{Statistical methods}

All statistical analyses were performed using SAS (version 8.1, Cary, NC, USA). Percentage reduction in tumour area following an initial cycle of carboplatin treatment was compared with tumour response after a second and third cycle by means of a two tailed, Student's $t$ test (alpha $=0.05)$. Percentage reduction in tumour area (per treatment) was then considered as a function of initial size of tumour using the initial largest basal diameter (0-4 disc diameters versus $>4$ disc diameters, where 1 disc diameter $(\mathrm{dd})=1.5 \mathrm{~mm})$, also using the Student's $t$ test $($ alpha $=0.05)$. Finally, percentage reduction in tumour area, per treatment, was stratified by tumour location/zone and analysed by ANOVA.

\section{RESULTS}

Thirty six tumours in 27 eyes of 21 patients were evaluated for response following 1-3 cycles of carboplatin. Table 1 summarises the patient demographics.

At baseline, 16 tumours measured 0-4 dd (44\%), 19 tumours measured $4-10 \mathrm{dd}(53 \%)$, and one tumour was more than 10 dd (3\%; fig 1). Following initial carboplatin treatment (mean follow up 22.0 days; 12-41), 34/36 tumours $(94.4 \%)$ showed a response to treatment and $2 / 36(5.6 \%)$ were stable. No tumours progressed. The mean reduction in area was $37.1 \%$ (median $37.0 \% ; 4.0 \%-76.7 \%$ ) and the mean reduction in largest basal diameter was $21.3 \%$ (median $21.0 \% ;-7.9 \%-52.5 \%)$. Tumour response following initial carboplatin treatment is stratified in figure 2.

Twenty of the original 36 tumours received a second cycle of carboplatin (mean follow up 26.1 days; range 20-35): 15

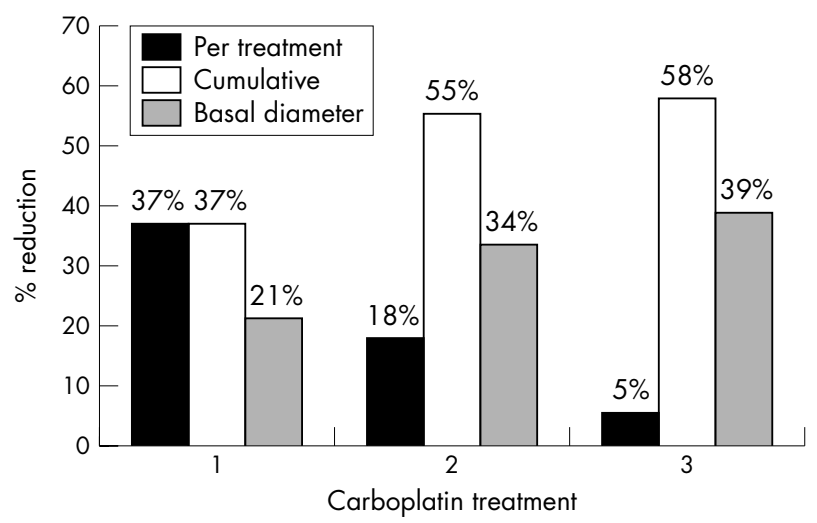

Figure 3 Mean reduction in tumour size. RET-CAM imaging software was used to measure tumour area and largest basal diameter, before and after each cycle of systemic carboplatin. 

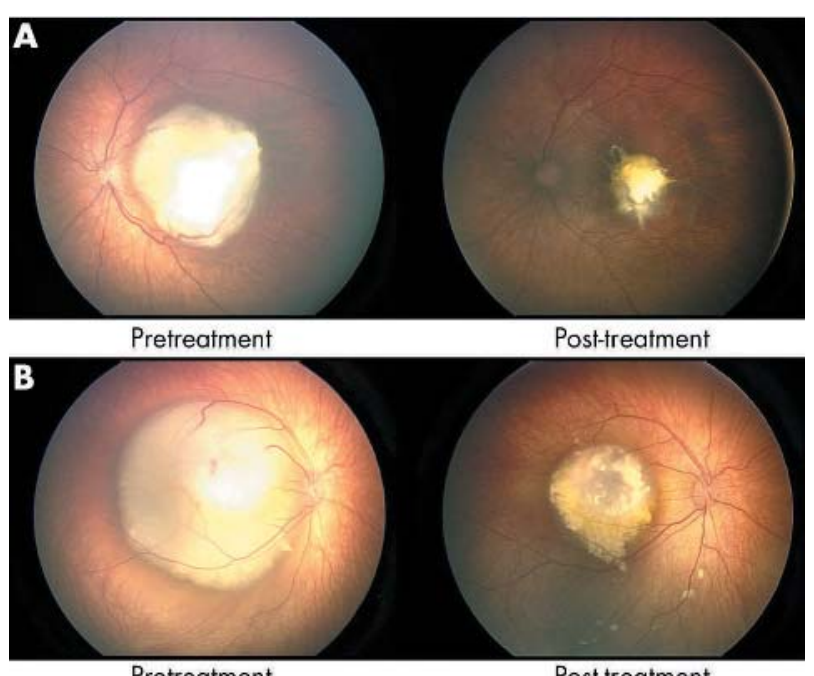

Pretreatment

Post-treatment

Figure 4 RET-CAM images of two solitary retinoblastomas: before and after treatment with primary carboplatin.

(75.0\%) were responsive to treatment and five $(25.0 \%)$ were stable. No tumours progressed. Mean reduction in area for the second treatment alone was $17.8 \%$ (median $15.3 \%$; range $-7.0 \%-49.7 \%$ ). The mean cumulative reduction in area after two treatments for the 20 tumours was $55.1 \%$ (median $56.2 \% ; 33.0 \%-74.5 \%)$, and the mean cumulative reduction in largest basal diameter was $33.6 \%$ (median 33.6\%; 10.9\%$53.2 \%)$.

Twelve tumours received a third cycle of carboplatin (mean follow up 21.8 days; 20-37): three tumours (25\%) were responsive, eight tumours $(67 \%)$ were stable, and one tumour showed progression. Mean reduction in area for the third treatment alone was $5.4 \%$ (median $7.2 \%$; $-17.7 \%-$ $20.6 \%)$. The mean cumulative reduction in area after three treatments was $58.1 \%$ (median $57.3 \%$; $34.8 \%-77.2 \%$ ), and the mean cumulative reduction in largest basal diameter was $38.8 \%$ (median 38.2\%; $19.1 \%-54.1 \%$ ). Figure 3 summarises the treatment specific and cumulative tumour responses.

The mean cumulative reduction in tumour area after both two and three cycles of systemic carboplatin was significantly greater than that achieved after one treatment $(p<0.01)$. Three cycles of carboplatin, however, did not result in a significantly greater response than two treatments $(\mathrm{p}>0.05)$. Percentage reduction in tumour area was not statistically related to initial tumour size (0-4 dd versus 4-10 dd; $\mathrm{p}=0.30$ ). Percentage reduction in tumour area was also not statistically related to tumour location $(\mathrm{p}>0.05)$. Tumour location and percentage reduction were compared following one cycle of carboplatin: macular versus macular-equator tumours $(p=0.10)$, macular versus equator-ora serrata $(\mathrm{p}=0.69)$, and macular-equator versus ora-serrata $(\mathrm{p}=0.27)$.

A proportion of the tumours that qualified for this study were in patients with intraocular retinoblastoma rigorously followed under a treatment protocol designed to evaluate patient and ocular survival after carboplatin chemotherapy. The observed side effects were minimal: mild blood count suppression was noted but was not clinically significant. No hearing loss specifically attributed to carboplatin treatment was observed, nor renal and/or hepatic toxicity (Dunkel, unpublished data, 2005).

Figure 4 shows RET-CAM images of two representative solitary tumours, each before and after treatment with primary carboplatin.

\section{DISCUSSION}

Previous studies have evaluated the response of intraocular retinoblastoma to chemoreduction using a variety of outcome variables and methodologies. The purpose of this study is to add to the existing literature measurements of tumour response after carboplatin chemotherapy. We chose to record the morphology of tumours in two dimensions before and after therapy, and felt this to be an accurate representation of response. Ocular and patient survival, and/or effectiveness of additional treatments for retinoblastoma were not assessed in this study.

Shields et al measured change in size of 54 tumours in 31 eyes following a 2 month regimen (two cycles) of triple agent chemotherapy (VEC). ${ }^{14}$ A $29 \%$ mean reduction in basal diameter and $40 \%$ mean reduction in thickness was reported after one cycle. A second cycle yielded a 35\% cumulative reduction in basal diameter and $49 \%$ in thickness. Each tumour was measured via echography and estimates by indirect ophthalmoscopy. Digital imaging was not used in reporting the data. The response rate to treatment was 100\%: approximately $50 \%$ of the tumours classified achieved a complete response after 2 months and the remaining 50\% achieved a partial response. No progressive disease was seen. In their analysis, the authors noted that larger tumours demonstrated a more dramatic response to treatment, but the percentage shrinkage was nearly identical between tumours $>8 \mathrm{~mm}$ thick versus tumours $<8 \mathrm{~mm}$ thick. Furthermore, they reported that in some patients, reduction in tumour size correlated with the location of the intraocular tumour: reduction correlated with increased distance of the tumour from the optic disc and foveola.

Sussman et al utilised RET-CAM imaging and echography to evaluate the effects of chemotherapy (VEC plus or minus cyclosporin) on RE group IV-V retinoblastomas. Their study did not purport to isolate the effects of systemic chemotherapy alone-patients received concurrent transpupillary diode laser and/or cryotherapy. The study objective was to compare the time course and extent of tumour reduction associated with this treatment regimen versus EBR in treating advanced intraocular retinoblastoma. Only the largest tumour in an eye was evaluated. The authors concluded that reduction in tumour volume was greatest in the first 2 months of therapy (68\% reduction from baseline after 1 month) and disappeared by 12 months. ${ }^{15}$

Finally, Demirci et al studied 10 eyes with intraocular retinoblastoma that had been enucleated after receiving an average of four cycles of VEC. Nine of the eyes were RE groups IV-V, and all 10 eyes had been enucleated for tumour recurrence as subretinal seeds and/or vitreous seeds (seven) or for vitreous haemorrhage (three). A $24 \%$ mean reduction of basal diameter and $34 \%$ in thickness of the main tumour in each eye was observed from pretreatment baseline to the time of enucleation. ${ }^{16}$

Of the 36 tumours we measured following exposure to carboplatin (1-3 cycles), 94\% responded. The remaining tumours were stable. One tumour progressed following the third cycle of carboplatin. These results are comparable with reported tumour regression following chemothermotherapy (CTT); whereby $96 \%$ of tumours responded after $1-6$ cycles. CTT consists of transpupillary thermotherapy (TTT) delivered to a tumour shortly after IV administration of carboplatin (plus or minus etoposide), with repeated TTT 8 days later. ${ }^{17}$

One cycle of carboplatin alone produced a $37.1 \%$ mean reduction in tumour area (fig $4 \mathrm{~A}$ and $4 \mathrm{~B}$ ). We found a continued reduction (17.8\%) in area after a second cycle. All tumours subjected to two cycles of carboplatin showed substantial cumulative regression (mean reduction in area $55.1 \%)$. When we looked at tumour response in terms of percentage reduction of the largest basal diameter, as other 
studies have done, we found a $21.3 \%$ mean decrease after one cycle of carboplatin. Two and three cycles of chemotherapy resulted in a cumulative reduction in largest basal diameter of $33.6 \%$ and $38.8 \%$, respectively. While variations in methodology limit comparison between individual studies, our results suggest that tumour response to treatment with a single agent (carboplatin) is comparable to that achieved in studies evaluating treatment with multiple agents.

Tumour response appeared to level off after two cycles of carboplatin. It is possible that maximal benefit of systemic chemotherapy is achieved during the first two cycles of treatment and that the residual mass represents treated tumour. It is also possible that our study selected for resistant tumours by focally treating tumours that responded more favourably to systemic carboplatin. Regardless, our observation is consistent with tumour reduction following EBR; it too appears to plateau, although the time to the plateau following EBR appears to be greater. ${ }^{15}$

Gombos et al, in an attempt to identify factors predictive of outcome, described tumour associated features of retinoblastomas successfully responding to triple agent chemotherapy (VEC). Their results suggested that small tumours $(<2 \mathrm{~mm}$ in basal diameter) were potentially less responsive to systemic chemotherapy. ${ }^{18}$ Furthermore, tumours in the macular region were more likely to be managed by systemic chemotherapy alone than tumours in the periphery; perhaps because disproportionate choroidal blood flow to the macular region facilitated greater drug delivery to centrally located tumours . Our study classified tumours on the basis of largest basal diameter in disc diameters $(0-4 \mathrm{dd}, 4-10 \mathrm{dd}$, and $>10$ $\mathrm{dd})$; size ranges reflecting the dimensions used for $\mathrm{RE}$ classification. Our analysis did not reveal a significant relation between initial tumour size and tumour response to treatment. However, there was a noticeable trend for each cycle of chemotherapy: larger tumours showed a greater percentage reduction in tumour area than smaller tumours for each treatment. Our analysis also did not reveal a correlation between the location of a given tumour and its percentage reduction in area following treatment with carboplatin. Given the information from previous studies by others, it is likely that our study was underpowered to detect differences in the percentage reduction across location.

Our results demonstrate quantifiable tumour chemoreduction following treatment with carboplatin. It appears comparable to the reported tumour response data following treatment with multi-agent chemotherapy; in terms of percentage reduction in largest basal diameter, percentage reduction in area, response rate, and early timing of maximum response. Our data suggest that, perhaps for some tumours, there is not a direct relation between the additional exposure to multiple chemotherapeutic agents and additional tumour reduction. We did not assess response to carboplatin treatment in terms of eye survival and/or eye event free survival, choosing not to introduce such variables as which focal technique is applied following chemoreduction, how often and how long it is applied, and the actual techniques used in applying the focal technique (which vary from centre to centre). Knowing that carboplatin alone will not cure the tumours despite a good initial response, complete analysis of these variables is necessary in order to (1) evaluate the overall success of a chemoreduction and focal therapy strategy for curing intraocular retinoblastoma, and (2) fully disclose to patients information regarding the risks and benefits of treatment choices.

\section{Authors' affiliations}

D H Abramson, K L Beaverson, T C Lee, I S Rollins, Ophthalmic Oncology Service, Department of Surgery, Memorial Sloan-Kettering Cancer Center, New York, NY, USA

D H Abramson, S D Lawrence, T C Lee, Department of Ophthalmology, New York Presbyterian Hospital-Weill Cornell Medical College, New York, NY, USA

K L Beaverson, Department of Medicine, Memorial Sloan-Kettering Cancer Center, New York, NY, USA

D H Abramson, I J Dunkel, Department of Pediatrics, Memorial SloanKettering Cancer Center, New York, NY, USA

Supported in part by the Fund for Ophthalmic Knowledge, New York, NY, USA.

Competing interests: none declared

\section{REFERENCES}

1 Chan HS, DeBoer G, Thiessen JJ, et al. Combining cyclosporine with chemotherapy controls intraocular retinoblastoma without requiring radiation. Clin Cancer Res 1996;2:1499-508

2 Rodriguez-Galindo C, Wilson MW, Haik BG, et al. Treatment of intraocular retinoblastoma with vincristine and carboplatin. J Clin Oncol 2003;21:2019-25.

3 Lee TC, Hayashi NI, Dunkel IJ, et al. New retinoblastoma tumor formation in children initially treated with systemic carboplatin. Ophthalmol 2003;1 10:10:1989-94.

4 Anagnoste SR, Scott IU, Murray TG, et al. Rhegmatogenous retinal detachment in retinoblastoma patients undergoing chemoreduction and cryotherapy. Am J Ophthalmol 2000;129:817-19.

5 Shields CL, Santos JA, Diniz W, et al. Thermotherapy for retinoblastoma. Arch Ophthalmol 1999;117:885-93.

6 Smith MA, Rubinstein L, Anderson JR, et al. Secondary leukemia or myelodysplastic syndrome after treatment with epipodophyllotoxins. J Clin Oncol 1999; 17:569-77.

7 Beck MN, Balmer A, Dessing C, et al. First-line chemotherapy with local treatment can prevent external-beam irradiation and enucleation in low-stage intraocular retinoblastoma. J Clin Oncol 2000;18:2881-7.

8 Kingston JE, Hungerford JL, Madreperla SA, et al. Results of combined chemotherapy and radiotherapy for advanced intraocular retinoblastoma. Arch Ophthalmol 1996;114:1339-43.

9 Gallie BL, Budning A, DeBoer G, et al. Chemotherapy with focal therapy can cure intraocular retinoblastoma without radiotherapy. Arch Ophthalmol 1996; 114:1321-8.

10 Friedman DL, Himelstein B, Shields CL, et al. Chemoreduction and local ophthalmic therapy for intraocular retinoblastoma. J Clin Oncol 2000; 18:12-17.

11 Murphree AL, Villablanca JG, Deegan III WF, et al. Chemotherapy plus local treatment in the management of intraocular retinoblastoma. Arch Ophthalmol 1996; 114:1348-56.

12 Shields CL, Shields JA, Needle M, et al. Combined chemoreduction and adjuvant treatment for intraocular retinoblastoma. Ophthalmology 1997; 104:2101-11.

13 Lumbroso L, Doz F, Levy C, et al. Diode laser thermotherapy and chemothermotherapy in the treatment of retinoblastoma. J Fr Ophtalmol. 2003;26: 2, 154-9.

14 Shields CL, De Potter P, Himelstein BP, et al. Chemoreduction in the initial management of intraocular retinoblastoma. Arch Ophthalmol 1996;114:1330-8.

15 Sussman DA, Escalona-Benz E, Benz MS, et al. Comparison of retinoblastoma reduction for chemotherapy vs external beam radiotherapy. Arch Ophthalmol 2003; 121:979-84.

16 Demirci H, Eagle RC, Shields CL, et al. Histopathologic findings in eyes with retinoblastoma treated only with chemoreduction. Arch Ophthalmol 2003;121:1125-31.

17 Lumbroso L, Doz F, Urbieta M, et al. Chemothermotherapy in the management of retinoblastoma. Ophthalmology 2002;109:1 130-6.

18 Gombos DS, Kelly A, Coen PG, et al. Retinoblastoma treated with primary chemotherapy alone: the significance of tumour size, location, and age. Br J Ophthalmol 2002;86:80-3. 